\title{
EXPERIMENTS REGARDING THE FABRICATION OF THE HIGHER STRENGTH STEELS PLATES
}

\author{
Marian BORDEI, Bogdan CARP \\ "Dunarea de Jos" University of Galati, Romania \\ e-mail: marian.bordei@ugal.ro
}

\begin{abstract}
The normalizing of ferritic-pearlitic steels is a heat treatment, which consist of reheating into the austenite and subsequent air-cooling. For greater thickness, e.g. $100 \mathrm{~mm}$, variations in the requirements may be permitted or required for particular applications but a reduction of the required impact energy is not allowed. The most important cote of the cost can be eliminated if the process normalizing rolling is applied.
\end{abstract}

KEYWORDS: higher strength steels, normalizing, structural applications

\section{Introduction}

The normalizing of ferritic-pearlitic steels is a heat treatment, which consist of re-heating into the austenite and subsequent air-cooling. Its names derive from the fact that any micro-structural changes caused by the production process, by overheating during forming or by hardening, will be reversed resulting in a homogeneous microstructure. Normalizing is the standard technology for steel castings and is often applied for forging and heavy rolled products, when high processing temperatures are common. As a result, the coarse microstructure will become more homogeneous and grain refined, which guarantees both higher strength and better toughness.

In the "normalizing rolled" process, the rather high finish rolling temperature of $1050{ }^{\circ} \mathrm{C}$ for normal processing of a $60 \mathrm{~mm}$ plate generates a rather coarse microstructure. If such a plate is processed in the lower austenite region at, for example, $880{ }^{\circ} \mathrm{C}$, its microstructure will be equivalent to that of steel in the normalized condition. Such finishing temperatures occur naturally with thinner gauges. For thicker material the rolling schedule has to be adjusted by introducing a delay time. In order to maintain the output of the mill, processing schedules have been developed, which allow for the simultaneous processing of more than one slab.

\section{Experimental study}

The LRS - E steel used for the structural applications is in conformity with LR Rules. The chemical analysis prescribed for the LRS-E steel sheets was tested and the results confirm equivalence with the LRS norms (Table 1).

The continuous cast slabs have been rolled at No. 2 heavy plate rolling mill and submitted to thermal treatment in the normalizing furnace. Subsequent to the normalizing treatment, the sheets were US controlled according to SEL 072-77 standard. The parameters of the casting are in Table 2 .

The results obtain of the mechanic tests and at the micro-structural analysis are presented in Tables 3 and the Figures 1-3.

Table 1. The chemical composition for the LRS-E steel

\begin{tabular}{|c|c|c|c|c|c|c|}
\hline $\mathrm{C}$ & $\mathrm{Mn}$ & $\mathrm{Si}$ & $\mathrm{P}$ & $\mathrm{S}$ & $\mathrm{Al}$ & $\mathrm{Nb}$ \\
\hline \multicolumn{7}{|c|}{$[\%]$} \\
\hline $0.15-0.18$ & $0.95-1.10$ & $0.15-0.30$ & max. 0.025 & max. 0.010 & $0.020-0.060$ & $0.030-0.050$ \\
\hline
\end{tabular}


THE ANNALS OF "DUNAREA DE JOS" UNIVERSITY OF GALATI

FASCICLE IX. METALLURGY AND MATERIALS SCIENCE

No. 2 - 2020, ISSN 2668-4748; e-ISSN 2668-4756

Article DOI: $\underline{\text { https://doi.org/10.35219/mms.2020.2.04 }}$

Table 2. The principal fabrication parameters for the LRS - E steel

\begin{tabular}{|c|c|c|c|}
\hline \multicolumn{2}{|c|}{ Tundish steel temperature } & ${ }^{\circ} \mathrm{C}$ & 1530 \\
\hline \multicolumn{2}{|c|}{ Casting time } & minutes & 59 \\
\hline \multicolumn{2}{|l|}{ Casting rate } & $\mathrm{m} / \mathrm{min}$ & 0.74 \\
\hline \multicolumn{2}{|l|}{ Slab size } & $\mathrm{mm}$ & $250 \times 1550$ \\
\hline \multicolumn{2}{|c|}{ Slab heating temperature } & ${ }^{\circ} \mathrm{C}$ & 1280 \\
\hline \multirow{2}{*}{ Slab/plate rolling temperature } & start & ${ }^{\circ} \mathrm{C}$ & 1180 \\
\hline & end & ${ }^{\circ} \mathrm{C}$ & 860 \\
\hline \multicolumn{2}{|l|}{ Plate thickness } & $\mathrm{mm}$ & 100 \\
\hline \multicolumn{2}{|c|}{ Normalizing temperature } & ${ }^{\circ} \mathrm{C}$ & 886 \\
\hline
\end{tabular}

Table 3. Steel grade: LRS E, sample 180-longitudinal tests, plate thickness $100 \mathrm{~mm}$

\begin{tabular}{|c|c|c|c|c|}
\hline Sample & $180-1 \mathrm{~L}$ & $180-2 \mathrm{~L}$ & $180-3 \mathrm{~L}$ & $180-4 \mathrm{~L}$ \\
\hline Section $(\mathrm{mm})$ & Ǿ 14 & Ǿ 14 & Ø 14 & Ǿ 14 \\
\hline Original gauge length Lo (mm) & 70 & 70 & 70 & 70 \\
\hline Final gauge length Lo (mm) & 91 & 91 & 91 & 90 \\
\hline Maximum force Fm (N) & 84500 & 85500 & 83400 & 84500 \\
\hline Flow force $\mathrm{F}(\mathrm{N})$ & 57500 & 60000 & 5500 & 58000 \\
\hline Proof stress at $0.2 \%\left(\mathrm{~N} / \mathrm{mm}^{2}\right)$ & 374 & 390 & 359 & 377 \\
\hline Tensile strength $\mathrm{R}_{\mathrm{m}}\left(\mathrm{N} / \mathrm{mm}^{2}\right)$ & 548 & 555 & 545 & 549 \\
\hline Total breaking elongation A (\%) & 30 & 30 & 30 & 28 \\
\hline $\begin{array}{c}\text { Charpy V-Notch test } 20^{\circ} \mathrm{C}(\mathrm{J}) \\
\text { Percentage crystallinity, \% } \\
\text { Lateral expansion }(\mathrm{mm})\end{array}$ & $\begin{array}{c}191-157-135 \\
91-80-77 \\
2.32-2.45- \\
2.63\end{array}$ & $\begin{array}{c}143-147-143 \\
80-80-85 \\
2.42-2.43- \\
2.45\end{array}$ & $\begin{array}{c}180-140-138 \\
80-88-80 \\
2.11-2.31- \\
2.63\end{array}$ & $\begin{array}{l}165-188-176 \\
81-90-86 \\
2.57-2.51- \\
2.55\end{array}$ \\
\hline $\begin{array}{l}\text { Charpy V-Notch test } 0^{\circ} \mathrm{C}(\mathrm{J}) \\
\text { Percentage crystallinity, } \% \\
\text { Lateral expansion }(\mathrm{mm})\end{array}$ & $\begin{array}{l}141-149-136 \\
85-85-77 \\
2.29-2.32- \\
2.32\end{array}$ & $\begin{array}{c}151-166-164 \\
85-81-80 \\
2.30-2.52- \\
2.40\end{array}$ & $\begin{array}{c}150-142-145 \\
851-80-85 \\
2.18-2.25- \\
2.34\end{array}$ & $\begin{array}{c}159-153-180 \\
80-85-88 \\
2.37-2.31- \\
2.23\end{array}$ \\
\hline $\begin{array}{c}\text { Charpy V-Notch test }-20{ }^{\circ} \mathrm{C}(\mathrm{J}) \\
\text { Percentage crystallinity, \% } \\
\text { Lateral expansion }(\mathrm{mm})\end{array}$ & $\begin{array}{c}124-117-115 \\
75-72-66 \\
1.98-2.08- \\
1.92 \\
\end{array}$ & $\begin{array}{c}120-110-114 \\
60-72-66 \\
2.02-1.96- \\
2.04\end{array}$ & $\begin{array}{c}124-111-125 \\
75-60-72 \\
1.96-2.09- \\
2.15\end{array}$ & $\begin{array}{c}139-144-134 \\
80-85-77 \\
2.42-2.36- \\
2.29\end{array}$ \\
\hline $\begin{array}{c}\text { Charpy V-Notch test }-40^{\circ} \mathrm{C}(\mathrm{J}) \\
\text { Percentage crystallinity, \% } \\
\text { Lateral expansion }(\mathrm{mm})\end{array}$ & $\begin{array}{c}43-62-70 \\
21-33-38 \\
1.56-1.39- \\
1.34 \\
\end{array}$ & $\begin{array}{c}62-72-43 \\
33-38-21 \\
1.39-1.56- \\
0.87\end{array}$ & $\begin{array}{c}51-47-51 \\
26-26-27 \\
0.62-0.65- \\
1.10 \\
\end{array}$ & $\begin{array}{l}47-52-81 \\
26-26-44 \\
0.55-0.65- \\
0.92 \\
\end{array}$ \\
\hline $\begin{array}{c}\text { Charpy V-Notch test }-60{ }^{\circ} \mathrm{C}(\mathrm{J}) \\
\text { Percentage crystallinity, \% } \\
\text { Lateral expansion }(\mathrm{mm})\end{array}$ & $\begin{array}{l}14-11-13 \\
11-12-11 \\
0.52-0.71- \\
0.42\end{array}$ & $\begin{array}{c}10-9-9 \\
11-11-12 \\
0.91-0.83- \\
0.67\end{array}$ & $\begin{array}{c}9-9-8 \\
11-12-11 \\
0.41-0.36- \\
0.73\end{array}$ & $\begin{array}{c}16-11-12 \\
17-11-12 \\
0.98-0.71- \\
0.65\end{array}$ \\
\hline Strain age Charpy tests $-40^{\circ} \mathrm{C}(\mathrm{J})$ & $8-7-6$ & $6-6-8$ & $7-18-10$ & $8-8-8$ \\
\hline Strain age Charpy tests $-60^{\circ} \mathrm{C}(\mathrm{J})$ & $5-5-6$ & $5-5-5$ & $6-5-5$ & $5-6-6$ \\
\hline Bend Tests & satisfy & satisfy & satisfy & satisfy \\
\hline
\end{tabular}




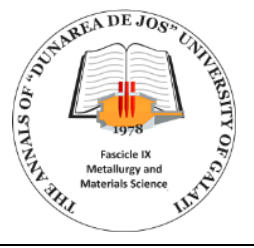

THE ANNALS OF "DUNAREA DE JOS" UNIVERSITY OF GALATI

FASCICLE IX. METALLURGY AND MATERIALS SCIENCE

$\mathrm{N}^{\circ} .2$ - 2020, ISSN 2668-4748; e-ISSN 2668-4756

Article DOI: https://doi.org/10.35219/mms.2020.2.04

\section{APPROVAL REPORT TEST TRANSITION CURVE E HEAT931275;L 178;TRANSVERSAL}

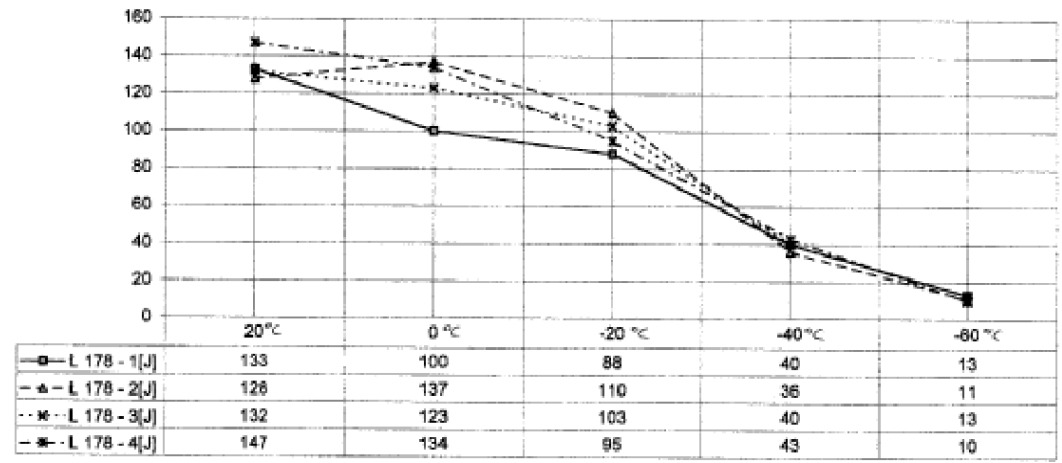

Fig. 1. Approval report test transition curve - transversal position

APPENDIX 6 - METALLOGRAPHIC ANALYSIS

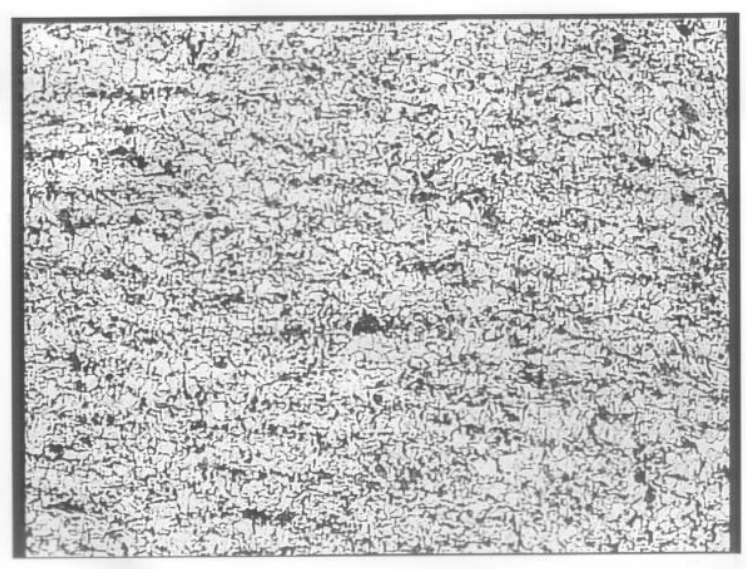

STEEL GRADE : LRS-E

HEAT NO : 931275

PLATE THICKNESS : $100 \mathrm{MM}$

STATE DELIVERY : N

SEAMPLE : L 178-1

LONGITUDINAL EDGE

FERRITE-PERLITE LINES STRUCTURE A2

GRAIN SIZE : 8-11

$100 \mathrm{X}$

Fig. 2. Metallographic analysis for longitudinal edge

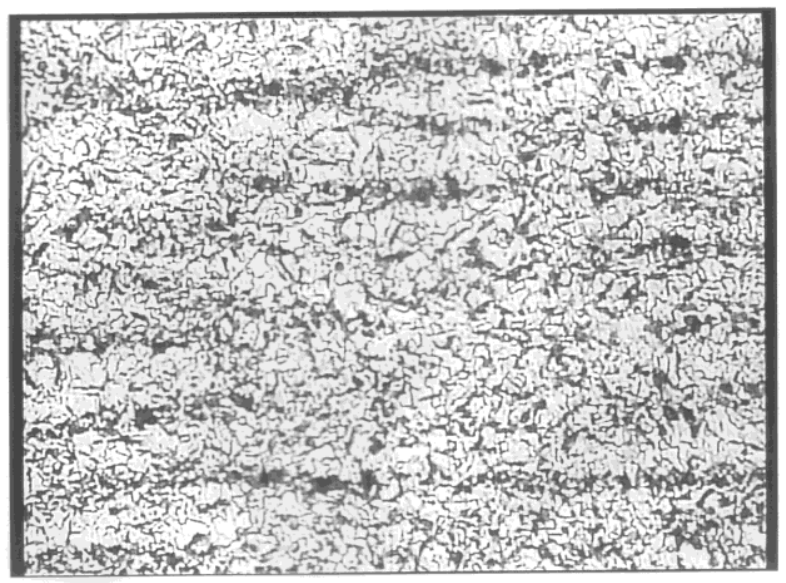

STEEL GRADE : LRS-E

HEAT NO : 931275

PLATE THICKNESS : $100 \mathrm{MM}$

STATE DELIVERY : N

SEAMPLE : L 178-1

LONGITUDINAL CENTRE

FERRITE-PERLITE LINES STRUCTURE A3

GRAIN SIZE : 7-10

$100 \mathrm{X}$

Fig. 3. Metallographic analysis for longitudinal centre 


\section{THE ANNALS OF "DUNAREA DE JOS" UNIVERSITY OF GALATI \\ FASCICLE IX. METALLURGY AND MATERIALS SCIENCE \\ $\mathrm{N}^{\circ} .2$ - 2020, ISSN 2668-4748; e-ISSN 2668-4756 \\ Article DOI: https://doi.org/10.35219/mms.2020.2.04}

\section{Conclusions}

In order to realize a program for extended a fabrication authorization of the higher strength steels plates for ship or other structural applications Grades $\mathrm{A}, \mathrm{B}, \mathrm{C}, \mathrm{D}$ and $\mathrm{E}$, on effectuated an researches and experimental series which consists in:

- chemical analysis;

- Baumann sulphur print;

- metallographic analysis;

- ultrasonic control.

For this analysis we on refer to the Romanian standards for the grain size, microstructures and the sulphur print.

The results obtained lead to the conclusion that the fabrication of the higher strength steels plates for ship or other structural applications, LRS-E3 type, is

accepted, because there are in conformity with the impose norms.

\section{References}

[1]. Weiss I., Dynamic Precipitation and Coarsening of $\mathrm{Nb}(\mathrm{CN})$ in hot compression of HSLA Steel, Metal. Trans. A, p. 403-410, March 1980.

[2]. Davenport A., The Recristallization of Austenite during the Hot Rolling of a Nb HSLS Steel, Hot Deformation of Austenite, AIME, 1977.

[3]. Brown E. L., Influence of Hot Rolling on Microstructure of Austenite, Hot Work. Form. Proc., Metals Soc., 1979.

[4]. Hernandez C., Fundamentos Metalurgicos de los Aceros Microaleados, Rev. Metal. CENIM, 6, p. 369-382, 1992.

[5]. ***, Guidelines for fabricating and processing plate steel, ArcelorMittal USA Plate production facilities Burns Harbor, IN Coatesville, PA Conshohocken, PA P +1 8009665352.

[6]. ***, Handbook of structural steel work, $4^{\text {th }}$ Edition, British Library Cataloguing-in-Publication, ISBN 185942133 4, 2002. 